\title{
Estudios de estabilidad transitoria en sistemas eléctricos industriales con generación propia interconectados con el sistema de transmisión
}

\section{Transient Stability Studies on Industrial Power Systems Interconnected to the Transmission Grid}

\author{
Calderón-Guizar J.G. \\ Gerencia de Análisis de Redes \\ Instituto de Investigaciones Eléctricas \\ E-mail:jgcg@iie.org.mx
}

(Recibido: junio de 2008; reevaluado: septiembre de 2009; aceptado: mayo de 2010)

\section{Resumen}

La continuidad en el suministro de energía eléctrica a procesos críticos de grandes complejos industriales, tales como plantas petroquímicas, procesadoras de gas, etc., es un aspecto de fundamental importancia para garantizar una operación adecuada de este tipo de complejos. Con el propósito de mejorar la confiabilidad en el suministro de energía a los procesos críticos, los sistemas eléctricos de estos complejos industriales, generalmente cuentan con generación propia. Sí la capacidad de generación instalada en los sistemas industriales es suficiente para satisfacer el total de la carga del complejo, la interconexión con el sistema de transmisión es utilizada principalmente como respaldo durante condiciones de emergencia. En este artículo se analiza el comportamiento dinámico de dos sistemas industriales con generación propia, diseñados para operar en forma aislada, interconectados entre sí, a través de un enlace de $115 \mathrm{kV}$ y con el sistema de transmisión. Los resultados obtenidos indican que el comportamiento de la frecuencia en estado "estable" en uno de los complejos, posterior a la pérdida del enlace con el sistema de transmisión, mejora de manera considerable cuando éstos operan de manera interconectada.

Descriptores: estabilidad transitoria, sistemas de potencia industriales, corte de carga.

\begin{abstract}
Aiming to ensure continuity in the electrical supply to critical processes, the electrical systems of large industrial plants, i.e. gas, and petrochemical industries usually posses local generation. Should the local generation be enough to satisfy the demand of the plant, then the interconnection with the public grid is commonly used as an important support for emergency conditions. This paper is concerned with the dynamical performance of two interconnected industrial power systems tied to the transmission system. Simulation results indicate that the frequency performance of one of the industrial plants, after the transients caused by the loss of the tie with the transmission system have died out, is greatly improved if both industrial plants remain connected after the disturbance.
\end{abstract}

Keywords: Stability, industrial power systems, load shedding. 
DOI: http://dx.doi.org/10.22201/fi.25940732e.2010.11n4.038

Estudios de estabilidad transitoria en sistemas eléctricos industriales con generación propia interconectados con el sistema de transmisión

\section{Introducción}

A pesar de que por razones diversas (Paine, 2000), (Shilling, 1997), los sistemas eléctricos de grandes complejos industriales, como lo son las refinerías, cementeras, papeleras, etc., usualmente cuentan con generación propia, el diseño y análisis de estos sistemas de potencia industriales se fundamenta principalmente en los estudios de corto circuito, flujos de carga, coordinación de protecciones, arranque de motores y arco eléctrico Smith (2007), Domen (1995), Dunki (1994). Sin embargo, los estudios de estabilidad transitoria son raramente requeridos durante el diseño de este tipo de sistemas, a pesar de que estos estudios resultan fundamentales para determinar la naturaleza de las medidas correctivas necesarias que permitan mitigar los efectos negativos en el comportamiento del sistema, causados por la ocurrencia de ciertos eventos durante la operación del mismo (Dunki, 1994). Algunas razones históricas por las que los estudios de estabilidad transitoria no han sido requeridos con la misma frecuencia que los de corto circuito o coordinación de protecciones en este tipo de sistemas son planteadas en Dunki (1994). La evolución natural y la integración de nuevos procesos en los grandes complejos industriales causa que sus necesidades de diferentes tipos de energía se incrementen. A raíz de la desregulación de la industria eléctrica, los complejos industriales con generación propia han optado por satisfacer sus necesidades incrementado de manera importante su capacidad de generación propia, ya que sus excedentes de energía pueden ser vendidos a las compañías comercializadoras de energía eléctrica (Paine, 2000). Adicionalmente, esta desregulación ha creado un gran interés por la interconexión con la red de transmisión de algunos sistemas eléctricos de potencia industriales, diseñados originalmente para operar en forma aislada (Rosales et al., 2000). Como resultado de esta tendencia en la evolución de los sistemas eléctricos de potencia de los grandes complejos industriales, los estudios de estabilidad transitoria resultan esenciales y no deben ser ignorados al evaluar la influencia, ya sea de la generación adicional en el sistema o de la interconexión con la red de transmisión en el comportamiento del sistema eléctrico del complejo (IEEE, 1997).

La estabilidad transitoria de un sistema eléctrico de potencia puede ser definida como la habilidad del sistema para permanecer en sincronismo cuando experimenta la ocurrencia de un disturbio severo (Kundur, 1994), los estudios de estabilidad transitoria tienen como objetivo fundamental el determinar la respuesta dinámica del sistema, durante y después de la ocurrencia de un "gran disturbio". En el caso de los sistema de potencia industriales, el objetivo de este tipo de estudios es evaluar el comportamiento dinámico del sistema ante la ocurrencia de disturbios tales como; corto circuitos en su red de distribución, pérdida súbita de grandes volúmenes de carga, pérdida de generación propia en el caso de sistemas interconectados con la red de transmisión, además de los eventos antes mencionados. Resultan también de interés, la pérdida de la interconexión con la red de transmisión y fallas en la red de transmisión eléctricamente cercanas al punto de interconexión, con el propósito de implementar acciones que permitan en lo posible, mantener la continuidad del suministro de energía eléctrica demandado por los diferentes procesos del complejo industrial.

En México, los sistemas eléctricos de potencia de los complejos de refinación fueron diseñados para operar en forma aislada de la red de transmisión (Rosales et al., 2000), con una capacidad de generación propia equivalente al doble de la carga del complejo, a fin de mantener la continuidad en el servicio. En el presente artículo, se evalúa el comportamiento dinámico de un sistema eléctrico de potencia industrial interconectado al sistema de transmisión para diferentes condiciones de operación.

\section{Estabilidad transitoria de sistemas eléctricos de potencia}

En general, la estabilidad en un sistema es una condición de equilibrio entre las fuerzas de oposición. Si un disturbio produce un desbalance sostenido entre las fuerzas de oposición, se presenta la condición de inestabilidad, la cual se manifiesta de formas diversas. En un Sistema Eléctrico de Potencia (SEP) ésta puede presentarse como pérdida de sincronismo u oscilaciones de magnitud creciente, por mencionar algunas. La naturaleza de los sistemas eléctricos de potencia es altamente no-lineal y operan en ambiente de cambio continuo. Algunos ejemplos son las variaciones normales en la carga, salidas de generadores y cambios en la topología de la red. Ante la ocurrencia de algún disturbio, la estabilidad del SEP es función de la magnitud del disturbio, la topología de la red de transmisión y de la condición de operación inicial del SEP. Los disturbios a los que se encuentra expuesto un SEP pueden ser clasificados de acuerdo a la magnitud de los mismos, como "disturbios de magnitud pequeña" y "disturbios severos o de gran magnitud", (los disturbios de magnitud pequeña, son las variaciones normales en la carga del sistema y la pérdida de generadores de pequeña capacidad, mientras 
DOI: http://dx.doi.org/10.22201/fi.25940732e.2010.11n4.038

Calderón-Guizar J.G.

que los disturbios de gran magnitud o severos, se consideran por la pérdida de generadores importantes y la ocurrencia de algún corto circuito en el sistema de transmisión). El problema de estabilidad en SEPs es un problema único, esto es, el SEP es estable o inestable. Sin embargo, como la inestabilidad puede manifestarse de diversas formas, resulta impráctico y poco útil el analizar la condición de estabilidad de un SEP como un problema único, debido a las grandes dimensiones y complejidad del modelo del SEP. En estudios de estabilidad, es necesario utilizar modelos simplificados con suficiente grado de detalle que permitan analizar únicamente determinadas manifestaciones de inestabilidad, usando las técnicas de análisis adecuadas. El problema de estabilidad transitoria, involucra el análisis de oscilaciones electromecánicas inherentes al SEP, por lo que la forma en que varía la potencia de salida de los generadores ante cambios en el ángulo de sus rotores es fundamental. En estado "estable" existe un equilibrio entre el par mecánico y el electromagnético de cada generador y su velocidad permanece constante, al ocurrir un disturbio, el equilibrio se pierde y se presentan aceleraciones y desaceleraciones de los rotores de los generadores. Así pues, si un generador gira a mayor velocidad que otro, la posición angular relativa de éste con respecto al generador que gira a menor velocidad se incrementará. Esta diferencia angular provoca que parte de la carga del generador que gira a menor velocidad sea transferida al generador que gira a mayor velocidad conforme a su relación "potencia-ángulo". Esta transferencia de carga tiende a "frenar" al generador que gira a mayor velocidad reduciendo la diferencia de velocidades entre ambos, y por consiguiente, la correspondiente diferencia angular.

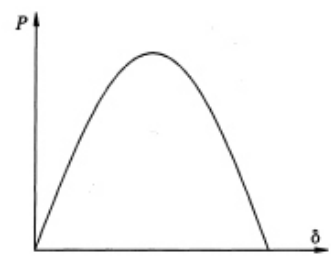

Figura 1. Relación potencia-ángulo

La relación "potencia-ángulo" indica que después de cierto límite, un incremento de la diferencia angular provocará una reducción en la potencia de salida, que a su vez, causará que la diferencia angular continúe incrementándose, lo cual conducirá a una condición de inestabilidad. Para una condición de operación dada, la estabilidad del SEP depende de si los pares "restauradores" causados por las desviaciones de las posiciones angulares de los rotores son "suficientes o no".
El problema de estabilidad transitoria se formula matemáticamente por un conjunto de ecuaciones diferenciales (las cuales describen la dinámica de los generadores y sus controles, sistema de excitación y sistema gobernador de velocidad turbina, así como las cargas de naturaleza dinámica) y otro conjunto de ecuaciones algebraicas (las cuales describen el comportamiento del sistema de transmisión/distribución al que se encuentran conectados los generadores). Normalmente las simulaciones de estabilidad transitoria se realizan resolviendo en el dominio del tiempo los sistemas de ecuaciones diferenciales y algebraicas representado por las ecuaciones 1 y 2.

$$
\begin{aligned}
& \stackrel{o}{X}=F(X, Y) \\
& O=G(X, Y)
\end{aligned}
$$

$\boldsymbol{X}$ representa a las variables de estado asociadas con los generadores y sus controles (ángulo del rotor, velocidad de los rotores, voltaje de excitación, potencia mecánica, etc.), así como a las de las cargas de naturaleza dinámica (motores de inducción). Mientras que $\boldsymbol{Y}$ representa a los voltajes/corrientes en los distintos nodos del sistema de transmisión/distribución. Al lector interesado en las técnicas para la solución de (1) y (2) se recomienda consultar el capítulo 13 de la referencia (Kundur, 1994).

\section{Descripción del sistema industrial interconectado}

El sistema de potencia industrial analizado es el resultado de la interconexión de dos sistemas eléctricos industriales con generación propia, diseñados inicialmente para operar en forma aislada entre sí y de sistema de transmisión. Por razones de confidencialidad, los nombres de los complejos industriales se han cambiado y únicamente se describen sus características relevantes para estudios de estabilidad transitoria. Cada uno de estos sistemas, tiene tres generadores de $30 \mathrm{MW}$, los correspondientes a la planta industrial identificada como "Planta $C$ " son todos de turbina de gas, mientras que de los correspondientes a la "Planta $N$ ", solo uno tiene turbina de gas y los otros dos de vapor. Los gobernadores de velocidad de los generadores de ambos complejos industriales operan en modo de "regulación", mientras los sistemas eléctricos de éstos operan interconectados con el sistema de transmisión. Cuando se pierde la interconexión con el sistema de transmisión, automáticamente los gobernadores de velocidad de los generadores de "Planta C" cambian de modo de operación "regulación" 
a modo "isócrono", los de "Planta $\boldsymbol{N}$ " continúan en modo "regulación".

En el presente trabajo, los dos escenarios operativos considerados, asumen que los complejos industriales suministran energía al sistema de transmisión. Los detalles de cada uno de los escenarios operativos son descritos a continuación:

- En este escenario operativo, denominado E.1. El complejo industrial $\mathbf{C}$ tiene en operación a sus tres generadores, satisface una demanda propia de 36 MW e inyecta $20 \mathrm{MW}$ al Sistema de Transmisión. Mientras que, el complejo industrial $\mathbf{N}$ tiene en operación únicamente dos de sus tres generadores, satisface una demanda propia de $48 \mathrm{MW}$ e inyecta $15 \mathrm{MW}$ al Sistema de Transmisión. Los generadores que se encuentran en operación en el complejo industrial $\mathbf{N}$ son aquellos con turbina de vapor.

- En este otro escenario operativo, denominado $\boldsymbol{E} .2$. El complejo industrial $\mathbf{C}$ tiene en operación únicamente a dos sus tres generadores, satisface una demanda propia de $36 \mathrm{MW}$ e inyecta $2 \mathrm{MW}$ al Sistema de Transmisión. Mientras que en el complejo industrial $\mathbf{N}$ se mantiene las mismas condiciones que las descritas en el escenario E.1.

Para ambos escenarios de operación, el interés principal radica en conocer el comportamiento de la frecuencia en ambos sistemas industriales, al perder de manera súbita el enlace con la red de transmisión considerando las siguientes dos condiciones de post-disturbio:

1. El enlace de $115 \mathrm{kV}$ entre los buses $\boldsymbol{H C}$ y $\boldsymbol{H}$ se mantiene cerrado, $\boldsymbol{N}$ y $\boldsymbol{C}$ operaran interconectados.

2. El enlace de $115 \mathrm{kV}$ entre los buses $(\boldsymbol{H C}$ y $\boldsymbol{H})$ se abre, $\boldsymbol{N}$ y $\boldsymbol{C}$ operaran de manera aislada.

Los resultados de las simulaciones realizadas indican que en ambos escenarios los sistemas $\boldsymbol{N}$ y $\boldsymbol{C}$, permanecen en sincronismo ante la pérdida súbita del enlace con el sistema de transmisión. Esto es, desde el punto de vista de estabilidad transitoria el sistema es estable como se deduce de las figuras 3, 4, 5 y 6 . En el caso, de las figuras 4 y 6 se observa que el comportamiento del ángulo de los rotores de los generadores del complejo $\boldsymbol{N}$ crece en forma monotónica, a diferencia del comportamiento que exhiben en las figuras 3 y 5 . Puesto que, la diferencia entre los ángulos de los rotores de los generadores del complejo $N$ se mantienen constantes, el sistema desde el punto de vista de estabilidad transitoria es estable (Kundur, 1994). Por otro lado, el comportamiento asociado con el ángulo de los rotores de los generadores del complejo $\mathbf{C}$ es oscilatorio amortiguado, similar al que exhiben en las figuras 3 y 5 . La razón de este comportamiento diferente, se debe a que los gobernadores de velocidad de los generadores del complejo $\boldsymbol{C}$ operan en modo "isócrono" y los del complejo $\boldsymbol{N}$ en modo "ación". Al perderse la interconexión con el sistema de transmisión, figuras 4 y 6 , el enlace entre los buses $\boldsymbol{H C}$

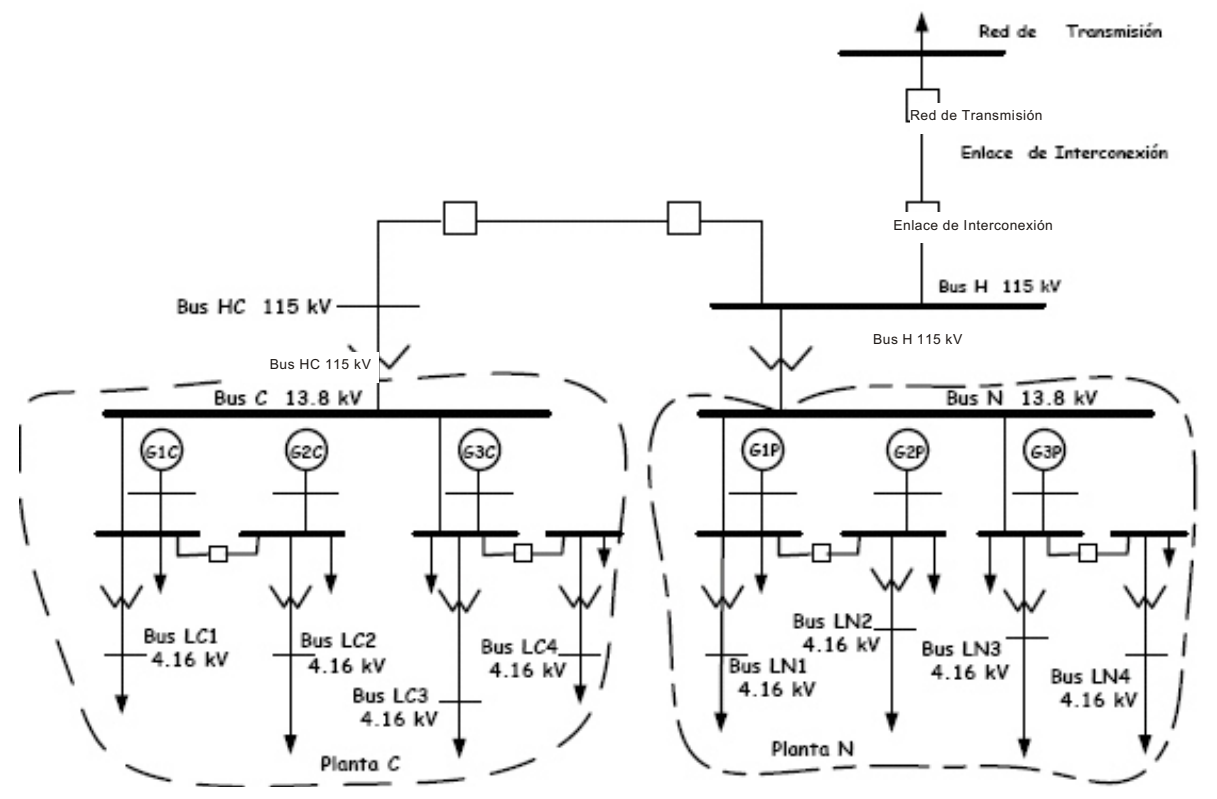

Figura 2. Diagrama unifilar del sistema de estudio 
DOI: http://dx.doi.org/10.22201/fi.25940732e.2010.11n4.038

Calderón-Guizar J.G.

y $\boldsymbol{H}$ se abre, quedando los sistemas eléctricos de los complejos $\boldsymbol{N}$ y $\boldsymbol{C}$, operando en forma aislada. Como consecuencia de esto, el modo "isócrono" de operación de los gobernadores de velocidad de los generadores de
$C$ no puede ejercer influencia alguna en larespuesta dinámica de los generadores de $\boldsymbol{N}$ y las respuestas de ambos sistemas resulta diferente.

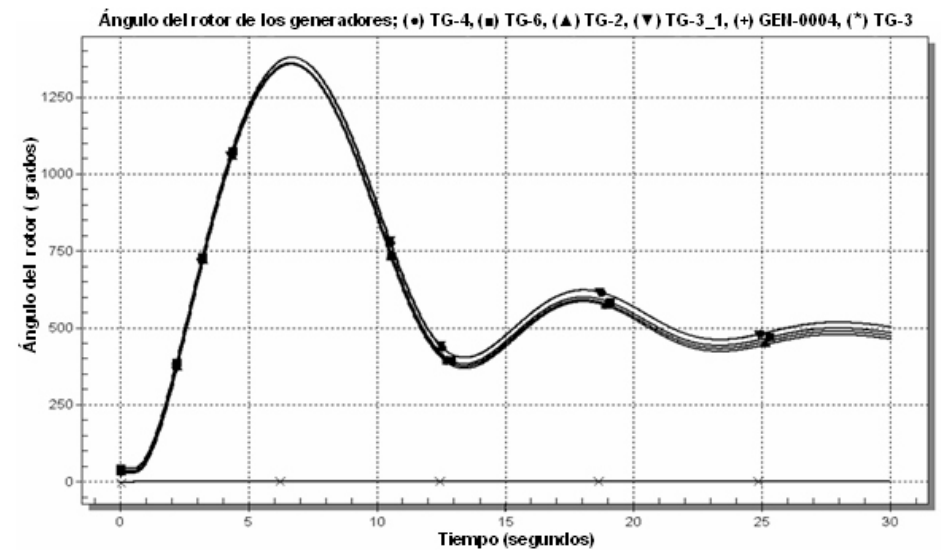

Figura 3. Ángulos de los rotores (E.1), sistemas de los complejos $\boldsymbol{N}$ y $\boldsymbol{C}$ interconectados

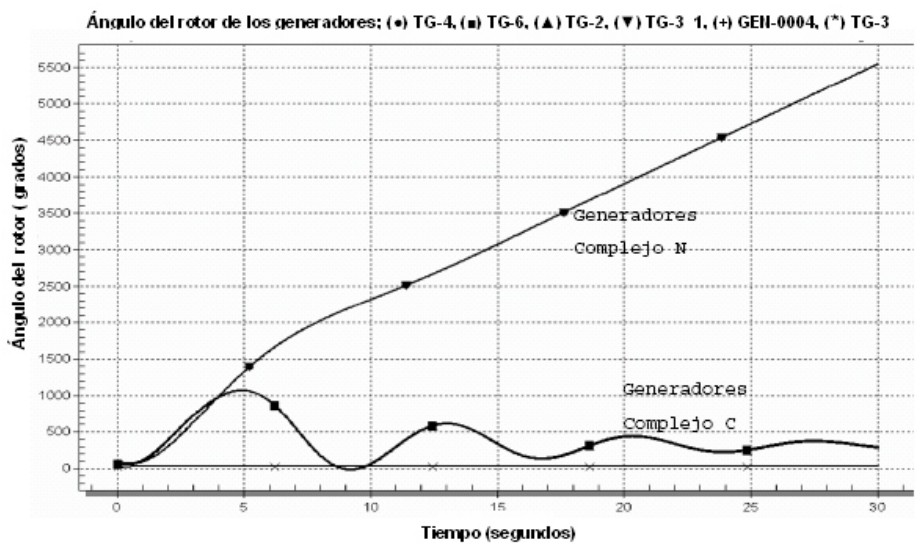

Figura 4. Ángulos de los rotores (E.1), sistemas de los complejos $\boldsymbol{N}$ y $\boldsymbol{C}$ aislados

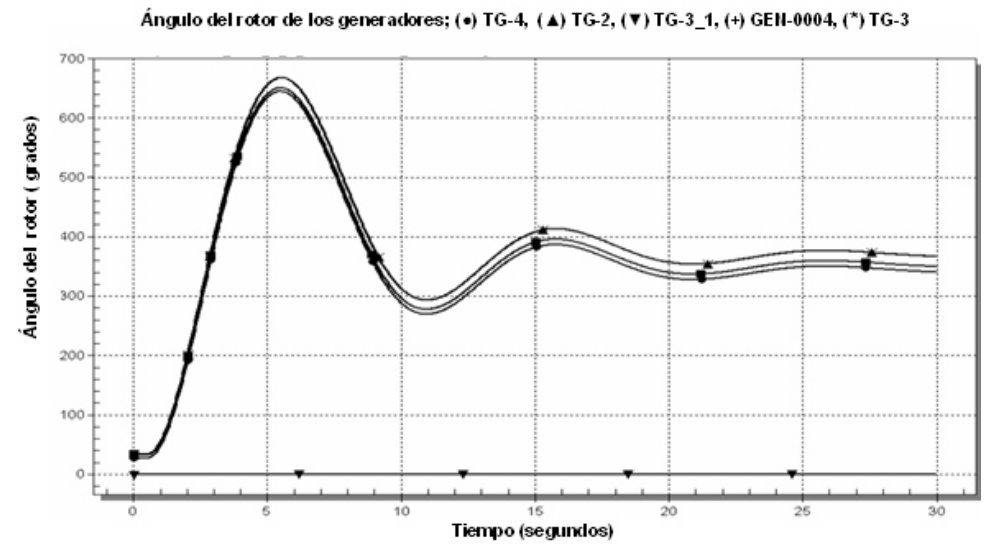

Figura 5. Ángulos de los rotores (E.2), sistemas de los complejos $\boldsymbol{N}$ y $\boldsymbol{C}$ interconectados 


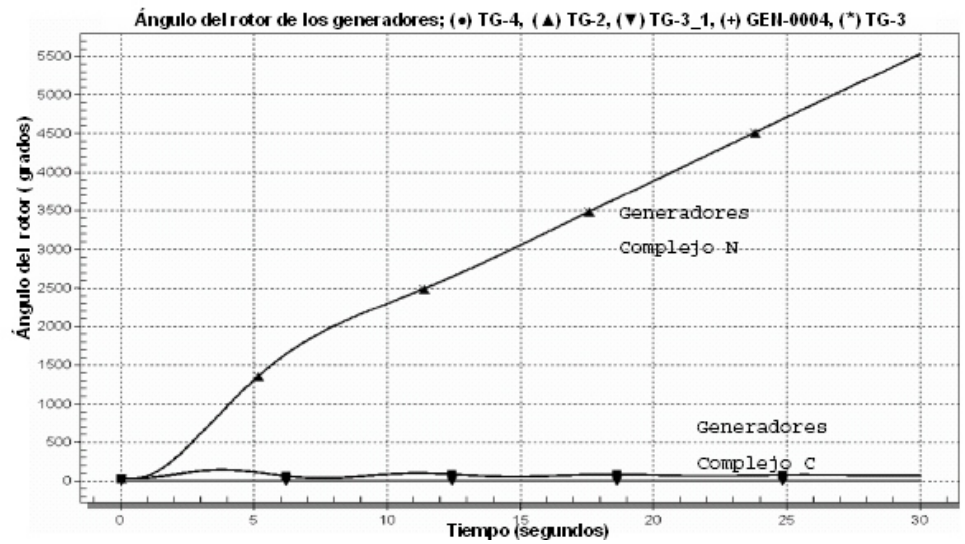

Figura 6. Ángulos de los rotores (E.2), sistemas de los complejos $\boldsymbol{N}$ y $\boldsymbol{C}$ aislados

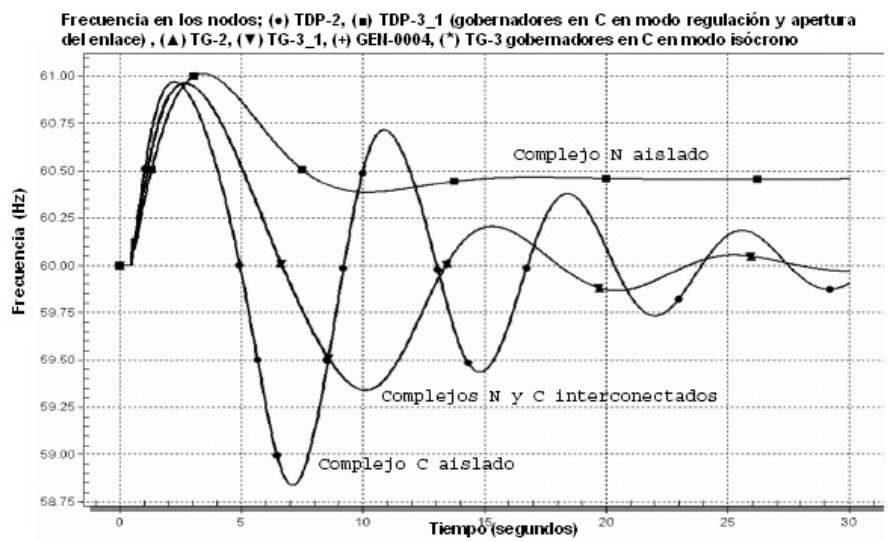

Figura 7. Comportamiento de la Frecuencia escenario E.1

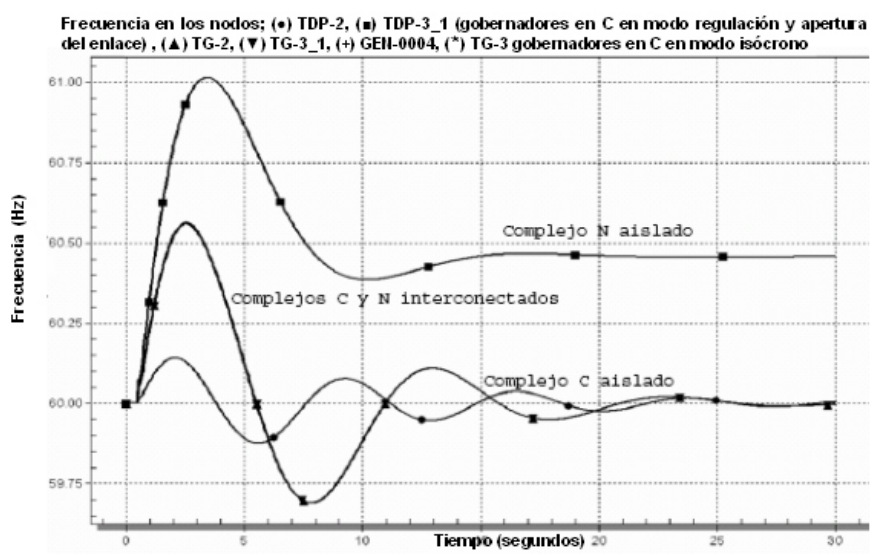

Figura 8. Comportamiento de la Frecuencia escenario E.2 
DOI: http://dx.doi.org/10.22201/fi.25940732e.2010.11n4.038

Calderón-Guizar J.G.

Se infiere también, que para el caso en que ambos complejos permanecen interconectados la frecuencia en el período de post-contingencia en ambos sistemas se restablece a su valor de pre-disturbio, mientras que cuando los complejos se separan, la frecuencia del sistema eléctrico del complejo $\boldsymbol{N}$ en el período de post-contingencia es mayor a su valor de pre-disturbio, en el caso del complejo $\mathbf{C}$ ésta se restablece a su valor de pre-disturbio como lo muestran la figuras 7 y 8 .

Se deduce del comportamiento de la frecuencia de los sistemas, mostrado en las figuras 7 y 8 , que resulta más conveniente para el sistema eléctrico del complejo $\boldsymbol{N}$ permanecer interconectado al del complejo $\boldsymbol{C}$, ante la apertura del enlace con el sistema de transmisión, ya que esta condición le permite restablecer su frecuencia de operación al valor de pre-disturbio, como consecuencia de la acción del modo de operación "isócrono" de los gobernadores de velocidad asociados con los generadores del complejo $\boldsymbol{C}$. Para el caso en que ambos sistemas se separan, la razón por la cual la frecuencia de operación del sistema del complejo $\boldsymbol{N}$ se estabiliza a un valor mayor que el de pre-disturbio, es que ninguno de los gobernadores de velocidad de los generadores de este complejo operan en modo "isócrono", por lo tanto, no se dispone de control alguno que al mismo tiempo satisfaga las nuevas condiciones de demanda y ajuste la frecuencia del sistema al valor de pre-disturbio.

\section{Conclusiones}

Los resultados mostrados en este artículo permiten concluir que para los escenarios considerados, a pesar de que la pérdida del enlace con el sistema de transmisión representa una pérdida de carga aproximada del 30\% de la condición inicial de carga en el escenario E.1, no es necesario disparar generación y que el mantener interconectados los sistemas eléctricos de los complejos, resulta benéfico para ambos, ya que la frecuencia de operación en la etapa de post-disturbio se reestablecerá al valor nominal de operación, debido a la acción del modo de operación "isócrono" de los gobernadores de velocidad de los generadores del complejo $\boldsymbol{C}$.

Se concluye también, que para los escenarios considerados, no es recomendable aislar a los sistemas eléctricos de los complejos, posterior a la pérdida de carga que causa la apertura del enlace con el sistema de transmisión, con el propósito de restablecer la frecuencia a su valor de pre-disturbio.

\section{Referencias}

Dommen-Mathew P., Kohler-Jeffery L. An Improved Model for Analyzing Industrial Power Systems. IEEE Trans. On Industry Applications, 31(5):1140-1146, 1995.

Dunki-Jacobs J.R., Davis-Chet E. An Argument and Procedure for Conceptual Power System Design Studies. IEEE Trans. On Industry Applications, 30(2):253-261, 1994.

IEEE. Recommended Practice for Industrial and Commercial Power Systems Analysis. IEEE Brown Book. IEEE Std 399-1997. 1997.

Kundur P. Power System Stability and Control. Mc Graw Hill. 1994.

Paine D.M. Increasing the Electrical Output of a Cogeneration Plant. IEEE Trans. On Industry Applications, 38(3): 726-735, 2002.

Rosales S.I., López V.J.J., García P.J., Robles P.E., García A.D., Guzmán C.B. Interconexión de centros industriales con la red eléctrica del servicio público. Boletín IIE, pp. 42-48, enero-febrero, 2000.

Shilling-Stanley R. Electrical Transient Stability and Underfrequency Load Shedding Analysis for a Large Pump Station. IEEE Trans. On Industry Applications, 33(1):194201, 1997.

Smith-Thomas P. Power System Studies for Cement plants. IEEE Industry Applications Magazine, pp. 56-65, July/August, 2007.

\section{Semblanza del autor}

Jorge Guillermo Calderón-Guizar. Es ingeniero industrial electricista (1983) por el Instituto Tecnológico de Morelia, obtuvo su maestría en ciencias en ingeniería eléctrica en 1986 en la Sección de Graduados de la ESIME-IPN. En 1995, el grado de doctor en filosofía ( $\mathrm{PhD}$ ) en ingeniería eléctrica en el Instituto de Ciencia y Tecnología de la Universidad de Manchester (UMIST) en el Reino Unido. Es investigador de la Gerencia de Análisis de Redes del Instituto de Investigaciones Eléctricas desde 1985. Senior Member del IEEE desde el 2001. Sus áreas de interés son el análisis dinámico y de estado estable de sistemas eléctricos de potencia de gran escala e industriales. 